\title{
Shortening of etching time of the dentin in primary teeth restorations: a randomized clinical trial
}

\author{
Cleber Paradzinski \\ CAVALHEIRO(a) iD \\ Pablo Soares de SOUZA ${ }^{(b)}$ iD \\ Diessica PEDROTTI(a) (iD \\ Luciano CASAGRANDE(c) iD \\ Thiago Machado ARDENGHI(d) iD \\ Rachel de Oliveira ROCHA(d) iD \\ Daniela Prócida RAGGIO(d) (ID) \\ Tathiane Larissa LENZI(c) \\ (a) Universidade Federal de Santa Maria - \\ UFSM, School of Dentistry, Dental Science \\ Graduate Program, Santa Maria, RS, Brazil. \\ (b) Universidade Federal de Santa Maria - \\ UFSM, School of Dentistry, Departament of \\ Stomatology, Santa Maria, RS, Brazil. \\ (c) Universidade Federal do Rio Grande do \\ Sul - UFRGS, School of Dentistry, Post- \\ Graduate Program in Pediatric Dentistry, \\ Porto Alegre, RS, Brazil. \\ (d) Universidade de São Paulo - USP, School of \\ Dentistry, Department of Orthodontics and \\ Pediatric Dentistry, São Paulo, SP, Brazil.
}

Declaration of Interests: The authors certify that they have no commercial or associative interest that represents a conflict of interest in connection with the manuscript.

\section{Corresponding Author:}

Tathiane Larissa Lenzi

E-mail: tathilenzi@hotmail.com

https://doi.org/10.1590/1807-3107bor-2020.vol34.0081

Submitted: March 5, 2020

Accepted for publication: May 18, 2020

Last revision: June 2, 2020

\begin{abstract}
The aim of this study was to investigate the influence of shortening of etching time for dentin on the restoration survival after selective carious tissue removal in primary molars. This two-arm randomized clinical trial included sixty-two subjects (5-8 year-old) and 100 primary molars presenting moderate dentin carious lesions on occlusal surface. The sample was randomly assigned into groups previously to adhesive application (Adper Single Bond 2; 3M ESPE): etching time recommended by manufacturer (15 s) or reduced (7 s). Resin composite (Filtek Bulk Fill Posterior Restorative; 3M ESPE) was inserted in a single increment for all restorations. Restorations were evaluated at 1, 6, 12 , and 18 months using FDI criteria. Survival estimates for restorations' longevity were evaluated with Kaplan-Meier method. Multivariate Cox regression analysis with shared frailty was used to assess the factors associated with failures $(\mathrm{p}<0.05)$. The etching time did not influence the restorations' survival (HR 0.35 95\%CI 0.11-1.12; $\mathrm{p}=0.06$ ). Mean estimated time of survival was 17.6 months $(95 \% \mathrm{CI}, 17.2-17.9)$. The survival rates at the 18-month follow-up were $75.7 \%$ and $91.4 \%$ (AFR: $16.9 \%$ and $5.7 \%$ ) when primary dentin was acid etched for 15 and $7 \mathrm{~s}$, respectively (log-rank $p=0.06$ ). In conclusion, the etching time for dentin did not influence the clinical behavior of adhesives restorations. However, there was a tendency for better clinical outcome when using etching time of $7 \mathrm{~s}$.
\end{abstract}

Keywords: Clinical Trial; Pediatric Dentistry; Acid Etching, Dental; Tooth, Deciduous.

\section{Introduction}

Resin composite has been widely used to restore decayed primary teeth because of its superior esthetics and lesser removal of sound tissue as compared to conventional treatments, thus allowing minimal intervention approaches such as selective carious tissue removal. Even though this material has shown satisfactory properties, a significant number of failures have been reported. ${ }^{1,2}$ Factors associated with children such as caries risk, ${ }^{3}$ oral hygiene, ${ }^{4}$ age, ${ }^{3,4}$ and behavior, as well as cavity-related features such as number of restored surfaces ${ }^{3,5}$ and presence of endodontic treatment ${ }^{1}$ could affect the restoration survival.

Although the choice of the type of composite material seems to have a minor effect on restoration survival, ${ }^{6}$ there is evidence of 
superior performance of etch-and-rinse adhesives in comparison with self-etch systems for restoring primary teeth ${ }^{7}$, being the most used by clinicians. However, it has been known that chemical ${ }^{8}$ and microstructural ${ }^{9}$ differences between primary and permanent dentin may jeopardize the adhesion in this substrate. ${ }^{10,11}$ Greater tubular density and larger diameter ${ }^{9}$ result in a reduced area of intertubular dentin available for bonding. Chemically, the lower mineral content ${ }^{8}$ reduces the buffering capacity and increases the reactivity of primary tooth dentin to acidic solutions. This is more critical while performing restorative procedures in cavity preparations involving residual carious tissue due to lesser mineral content in this substrate. ${ }^{12}$

Deeper demineralization of the dentinal substrate and subsequent incomplete penetration of resin monomers into the demineralized area results in a non-impregnated zone at the bottom of the hybrid layer, which creates sites more prone to degradation over time. ${ }^{13}$ Thus, a previous in vitro study ${ }^{14}$ stated that acid etching for half the time recommended by the manufacturer improves the bond stability with sound and carious primary dentin when etch-and-rinse adhesives are employed. Unfortunately, there is a lack of clinical evidence for the same.

Since randomized clinical trials provide the necessary support to clinicians in an evidence-based decision-making process, the aim of this study was to investigate the influence of shortening of etching time for dentin on the restoration survival after selective carious tissue removal in primary molars.

\section{Methodology}

\section{Study design and ethical concern}

This was a two-arm, parallel, randomized clinical trial that followed the CONSORT (Consolidated Standards of Reporting Trials) statement, and the study has been registered on the website www.clinicaltrials.gov (\#NCT02969538). The local Ethics Committee on Investigations Involving Human Subjects of the Federal University of Santa Maria reviewed and approved the protocol and consent form for this study (protocol 1.320.844). Written informed consent was obtained from the guardians of the participants prior to starting the treatment.

The study was carried out in the Pediatric Dentistry Clinic of the School of Dentistry, Federal University of Santa Maria, Santa Maria, Rio Grande do Sul, Brazil, from April 2016 to October 2018. The participants and their guardians received detailed information about the study, but they were not aware of the treatment provided by the specific restoration under evaluation.

\section{Sample calculation}

To perform the sample size calculation, the expected success rate of occlusal resin composite restorations in primary molars was considered $95 \%$ in 18 month $^{15}$. It was considered that a clinically significant difference was $15 \%$ in the success rate between the groups. Therefore, considering a significance level of 0.05 and a power of 0.80 , using a one-tailed test for non-inferiority studies, with a $20 \%$ increase due to a possible sample loss and 30\% by cluster of more than one tooth per children, we reached the final rounded number of 48 teeth per group, resulting in 96 teeth in total.

\section{Sample selection}

A total of 130 children (aged 5-8 years) were examined by two dentists (R.O.R. and T.M.A.) check the inclusion and exclusion criteria (Figure 1). The participants were recruited in the order that they attended the screening appointment. The clinical evaluations were performed using a plain mouth mirror and a ballpoint probe (Hu-Friedy Manufacturing Co., Chicago, USA). Children presenting good general health with cooperative behavior that did not refuse with the completion of clinical procedure with at least one primary molar with a moderate occlusal dentinal carious lesion, and with antagonist tooth were included in the study. The depth of the lesions was confirmed by bitewing radiographic examinations, i.e., the caries lesions should involve the middle third of dentin radiographically. Moreover, the inclusion criteria also required that the participants presented teeth with the following clinical and radiographic features: absence of sensitivity and/or spontaneous pain, swelling, fistula, and mobility incompatible with the root resorption stage, absence of any radiographic signs suggesting pulp necrosis. Participants requiring 
any other dental treatment were referred to the Dental School of the University. All individuals received dietary and oral hygiene instructions. Based on the pre-established criteria, 62 patients were selected for this study (Figure 1).

\section{Training and calibration}

One examiner (D.P.) underwent $8 \mathrm{~h}$ of specific training session involving theoretical explanations, discussion, and assessment of 20 representative photographs of each score of the World Dental
Federation (FDI) criteria. ${ }^{16}$ After these procedures, the examiner evaluated restorations in 10 children and repeated the evaluations after two weeks to determine intra-examiner agreement. A benchmark examiner (T.L.L.) also performed the examinations to calculate the inter-examiner reliability. The operator (C.P.C.) was a graduate dental student, specialist in Pediatric Dentistry, and underwent training to perform the restorations in children ( $10 \%$ of the sample) not included in this study. The operator was assisted by a dental assistant (P.S.S.).

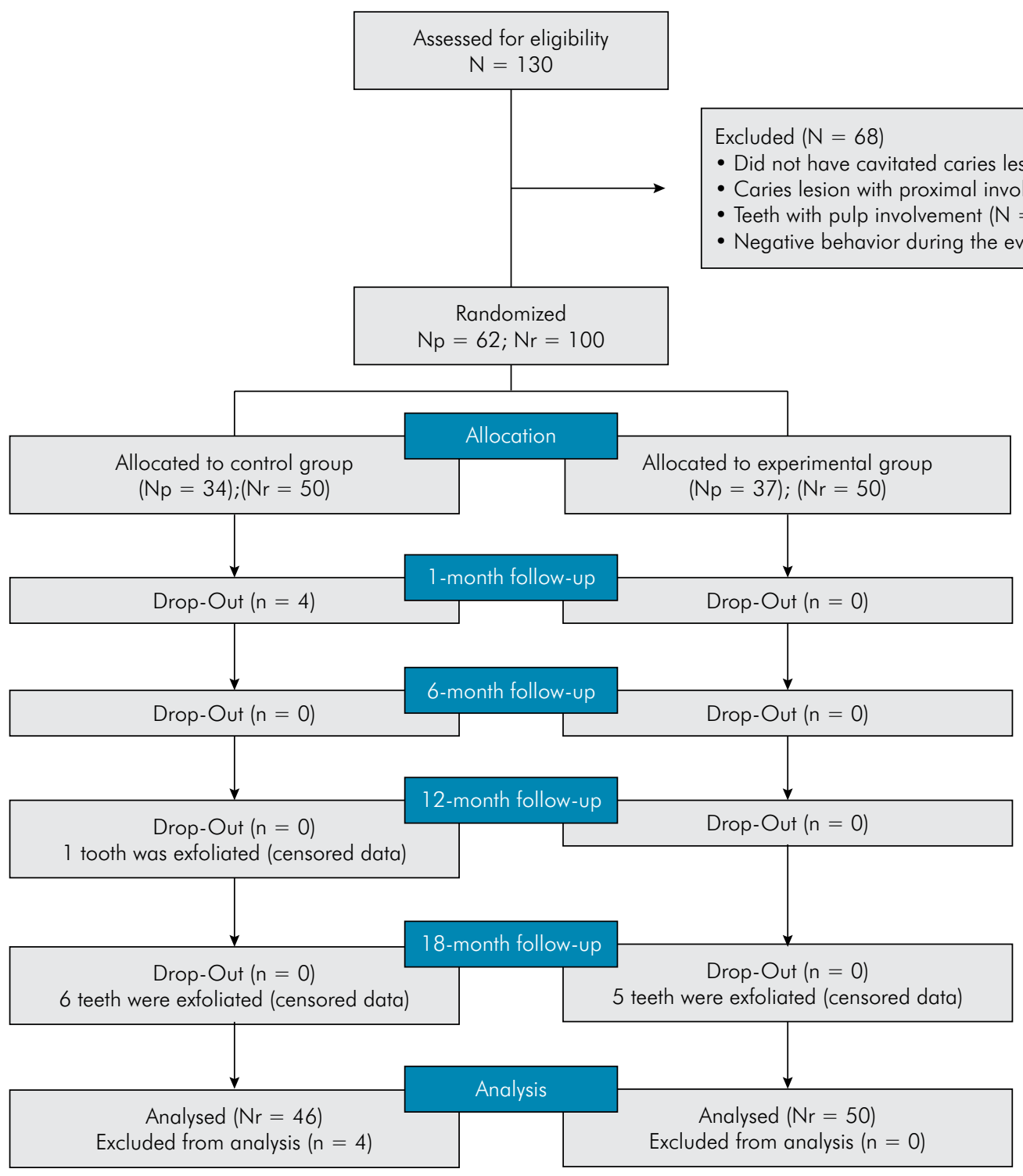

Np: number of patients; Nr: number of restorations.

Figure 1. CONSORT flowchart of the participants' progress through the trial phases. 


\section{Randomization, allocation and blinding}

The randomization was performed by a staff member (D.P.R.) who was not involved in any of the clinical trial phases. Teeth were randomly allocated to each group according to a sequence obtained using the appropriate software (Random Allocation 1.0, Isfahan, Iran). The allocation concealment was guaranteed by the use of opaque and numbered individual envelopes. The envelopes were opened after the selective carious tissue removal procedure. When the same patient had more than one tooth included, the order of the treated teeth was based on tooth number, being the tooth with the lowest tooth number received the treatment first. Although the operator was not blinded to group assignment when performing the interventions, the participants were blinded. Furthermore, the examiner, who did not take part in the restoration phase, was blinded.

\section{Treatment procedures}

The two groups of interventions were defined according to the acid etching time of primary dentin: recommended by manufacturer (15s) or reduced (7s). After dental prophylaxis, local anesthesia and rubber dam isolation were performed. Spoon excavators were used for selective carious tissue removal up to firm dentin $^{17}$, and carbide burs at low speed ( $\mathrm{N}^{\circ}$. 2) were used for complete carious tissue removal from the cavosurface margins and all lateral walls. Dentinal carious lesions were accessed when necessary using a spherical diamond bur ( $\mathrm{N}^{\circ}$. 1011; KG Sorensen, São Paulo, Brazil) operated at high speed under water-cooling. Visual and tactile criteria were used to guide selective carious tissue removal up to firm dentin on pulpal floor. Excavation was stopped when hard and dried dentin with a leathery consistency (resistant to spoon excavator) was reached. ${ }^{17}$

After removing carious tissue, each cavity was washed with water spray until it was visually clean and then dried. The margins of the enamel cavity of the teeth of both groups were etched with 35\% phosphoric acid gel (EtchantScotch Bond, 3M ESPE, St. Paul, USA) for $15 \mathrm{~s}$, while the dentin was acid etched for $15 \mathrm{~s}$ or $7 \mathrm{~s}$. In the reduced etching time group, phosphoric acid was firstly applied for $8 \mathrm{~s}$ on enamel and then applied on dentin for $7 \mathrm{~s}$, totalizing $15 \mathrm{~s}$ of enamel acid etching. The etching time was measured using a digital stopwatch. Cavities were flushed with air/water spray, and dried with sterilized absorbent papers leaving a slightly glistening appearance. Two layers of the adhesive system (Adper Single Bond 2; 3M ESPE, St. Paul, USA) were actively applied on the entire preparation in both groups according to the manufacturer recommendations. This was followed by gentle air thinning for $5 \mathrm{~s}$ and light curing (Radii-cal; SDI, Victoria, AUS) for $10 \mathrm{~s}$. Resin composite (Filtek Bulk Fill Posterior Restorative, shade A1; 3M ESPE, St. Paul, USA) was inserted as a single increment of approximately $4 \mathrm{~mm}$ and light cured for $20 \mathrm{~s}$ on each surface of the tooth. The occlusion was checked by using articulating paper, and the restorations were finished immediately using fine diamond burs (KG Sorensen, São Paulo, Brazil). Polishing was performed using rubber points one week after restoration (Astropol, Ivoclar Vivadent, Schaan, Liechtenstein).

\section{Evaluation}

The restorations were evaluated according to the FDI criteria ${ }^{16}$ by a trained and calibrated evaluator (D.P.) after 1, 6, 12, and 18 months of the restoration. The patients were recalled for follow-up by telephone contact, and the evaluations were performed in dental chair using artificial dental light, triple syringe, plain mouth mirror and probe. All parameters during the evaluation were recorded using a standardized manual case report form. FDI criteria ${ }^{16}$ were categorized into three groups: esthetic (marginal staining), functional (fracture and marginal adaptation), and biological (caries recurrence). The worst score among all evaluated parameters was considered. Each criterion of FDI was evaluated for five scores, of which three scores indicated acceptable restorations (1: clinically very good; 2 : clinically good; 3 : clinically sufficient/satisfactory) and two scores indicated non-acceptable restorations (4: clinically unsatisfactory, repairable restoration; 5: clinically poor, restoration replacement required). The restorations were recorded as failed if they scored 4 or 5 of the FDI criteria.

\section{Statistical analysis}

Distribution of success rates of restorations was calculated according to the independent variables of 
the following demographic and clinical characteristics: gender (male/female), tooth type (first/second molar), arch (upper/lower), dentin etching time (15 s/7 s), presence of visible plaque (no/yes), and gingival bleeding (no/yes) at the site of the restoration after follow-up. Restoration longevity was assessed by Kaplan-Meier estimator. Differences on survival rates according to the clinical variables were tested by the log-rank test and the significance level was set at $5 \%$. The annual failure rate (AFR) was calculated using the formula: $(1-y) z=(1-x)$, where " $y$ " is the mean AFR and " $x$ " is the total failure rate at " $z$ " years.

Survival estimates for restorations' longevity were evaluated using the Kaplan-Meier method. Multivariate Cox regression models with shared frailty were performed to identify factors associated with failure of the restorations. These models consider that observations within the same group (the patient) are correlated, sharing the same frailty, and are analogous to multilevel regression models with random effects. Test of proportional-hazards assumption was performed for each independent variable before including them in the regression analysis. Hazard ratios (HR) and their respective 95\% confidence intervals (CI) were obtained. A backward stepwise procedure was used to select covariates in the fitting of the model. Only those variables presenting $p<0.20$ were selected for inclusion in the final model. The significance level was set at $5 \%$. Kappa statistics was used to test the intra-examiner and interexaminer agreement. Data analyses were performed using the STATA 12.0 software (Stata Corp., College Station, Texas, USA).

\section{Results}

The sample comprised of 100 restorations in 62 subjects ( 24 male and 38 female) with an average age of 7.1 years ( \pm 1.5 years), presenting a decayed, missing, and filled primary teeth ( $\mathrm{dmft}$ ) index mean of $5.3 \pm 1.7$. Household income ranged between one and two Brazilian minimum wages, which nearly correspond to $\$ 273$ to $\$ 564$ during the period of the study. The follow-up period ranged from 1 to 18 months. Dropouts included four participants (6.4\%) and four (4\%) restorations. Physiological exfoliation was seen for 1 tooth and 11 teeth at 12 and 18 months, respectively. Finally, 84 restorations were evaluated after follow-up (Figure 1).

The distribution of the restorations according to the individual and tooth-level variables is shown in Table 1. Restorations performed in second molars were more common than those placed in first molars, and there were more restorations in the lower arch than in the upper arch, irrespective of the intervention group. Most restorations had visible plaque on the site after follow-up. Restorations performed after $15 \mathrm{~s}$ and $7 \mathrm{~s}$ of dentin etching time were more frequent in male and female, respectively. Table 2 shows descriptive crude and adjusted Cox regression analysis for failure of restorations according to independent variables. Individual and tooth-level variables did not affect the longevity of the restorations ( $p>0.05$ ). However, using Hazard Ratio (HR) values, the shortening of the acid etching time $(7 \mathrm{~s})$ for dentin presented an HR of $65 \%(p=0.06)$ in comparison with the acid etching time recommended by the manufacturer (15 s). Mean estimated time of survival was 17.6 months (95\%CI: 17.2-17.9). Estimated survival rates of the restorations were $100 \%, 97.9 \%, 94.8 \%$, and $84 \%$ at $1,6,12$, and 18 months of follow-up evaluations, respectively (Figure 2). The survival rates at the 18-month follow-up were $75.7 \%$ and $91.4 \%$ (AFR: $16.9 \%$ and $5.7 \%$ ) when primary dentin was acid etched for 15 and $7 \mathrm{~s}$, respectively (long-rank $\mathrm{p}=0.06$ ). The distribution of the restorations, according to the FDI criteria, is summarized in Table 3 . Ten restorations failed when dentin was acid etched by 15s, and 4 failures occurred when using reduced acid etching. Marginal adaptation and fracture were the main reasons for failures of resin composite restorations (scores 4 and 5). Additionally, two failures due marginal staining were observed when dentin was acid etched by $15 \mathrm{~s}$. The weighted kappa values for intra and interexaminer agreement were 0.92 and 0.89 , respectively.

\section{Discussion}

Dentin etching is a crucial step to attain effective bonding. Application time, concentration, composition, and $\mathrm{pH}$ of acid solution affect the depth of intertubular dentin demineralization. ${ }^{10}$ Furthermore, the substrate 
Table 1. Distribution of individual and tooth-related variables according to the treatment ( $\mathrm{n}=96$ restorations).

\begin{tabular}{lcc}
\hline $\begin{array}{l}\text { Variables } \\
\text { Gender }\end{array}$ & Dentin etching time $(15 \mathrm{~s})$ & Dentin etching time $(7 \mathrm{~s})$ \\
Female & $20(43.5)$ & $30(60.0)$ \\
Male & $26(56.5)$ & $20(40.0)$ \\
$\begin{array}{l}\text { Teeth } \\
1^{\text {st }} \text { molar }\end{array}$ & $14(30.4)$ & $16(32.0)$ \\
$2^{\text {nd }}$ molar & $32(69.6)$ & $34(68.0)$ \\
Arch & $16(34.8)$ & $18(36.0)$ \\
Upper & $30(65.2)$ & $32(64.0)$ \\
Lower & $25(54.3)$ & $26(52.0)$ \\
Gingival bleeding on the site & $24(48.0)$ \\
No & $21(45.7)$ & $14(28.0)$ \\
Yes & $9(19.6)$ & $36(72.0)$ \\
Visible plaque on the site & \\
No & $37(80.4)$ & \\
Yes &
\end{tabular}

type may interfere with bonding effectiveness, and consequently affect the longevity of the restoration.

Primary tooth dentin has greater tubular density and larger diameter than that of permanent tooth dentin'. Since the penetration of acids occurs primarily along the tubules, it could be possible that denser and larger diameter tubules could result in deeper penetration of the acidic conditioner. Primary tooth dentin also seems to be more reactive to acid etching due to a reduced degree of mineralization . $^{8}$

Nowadays, selective carious tissue removal to firm dentin is the treatment of choice for moderately deep cavitated dentinal lesions in order to maximize the longevity of the restorations by sufficient removal of soft dentin ${ }^{17}$. Although enough infected dentin is removed, the demineralized dentin, which can be remineralized, is maintained on the pulpal floor. This substrate presents a higher number of porosities in intertubular dentin, facilitating the diffusion of the inorganic conditioners, while the buffering action of the mineral phase of dentin is compromised by reduced mineral content. As consequence, a deeper demineralized layer forms thicker hybrid layers than those produced in sound dentin ${ }^{18}$. Therefore, an acid etching time reduced by approximately $50 \%$ for etch-and-rinse adhesive systems could reduce the occurrence of an unprotected dentin zone along the bottom of hybrid layers, mainly in a carious substrate. ${ }^{14}$

It has been demonstrated that shortening of the dentin acid etching time is not detrimental to immediate bond strength, ${ }^{19}$ and reduces the degradation of bond strength created by etch-and-rinse adhesive systems in

Table 2. Descriptive crude and adjusted (HR;95\%Cl) Cox regression analysis for failure of restorations according to the independent variables.

\begin{tabular}{|c|c|c|c|c|c|c|}
\hline Variables & $\mathrm{HR}_{\text {crude }}(95 \% \mathrm{Cl})$ & $\mathrm{p}$-value & $\mathrm{HR}_{\text {adiusted }}(95 \% \mathrm{Cl})$ & $\mathrm{p}$-value & Success (\%) & Failure (\%) \\
\hline Gender & & 0.94 & & & & \\
\hline Female & 1 & & & & $43(86.0)$ & $7(14.0)$ \\
\hline Male & $1.04(0.37-2.97)$ & & & & 39 (84.8) & $7(15.2)$ \\
\hline Teeth & & 0.06 & & 0.08 & & \\
\hline $1^{\text {st }}$ molar & 1 & & 1 & & $22(73.3)$ & $8(26.7)$ \\
\hline $2^{\text {nd }}$ molar & $0.35(0.12-1.00)$ & & $0.38(0.13-1.12)$ & & $60(90.9)$ & $6(9.1)$ \\
\hline Arch & & 0.71 & & & & \\
\hline Upper & 1 & & & & $30(88.2)$ & $4(11.8)$ \\
\hline Lower & $1.25(0.39-3.97)$ & & & & 52 (83.9) & $10(16.1)$ \\
\hline Gingival bleeding on the site & & 0.22 & & 0.20 & & \\
\hline No & 1 & & 1 & & $47(90.4)$ & $5(9.6)$ \\
\hline Yes & $1.97(0.66-5.88)$ & & $1.87(0.62-5.62)$ & & $35(79.5)$ & $9(20.5)$ \\
\hline Visible plaque on the site & & 0.46 & & & & \\
\hline No & 1 & & & & 19 (82.6) & $4(17.4)$ \\
\hline Yes & $0.64(0.20-2.05)$ & & & & $63(86.3)$ & $10(13.7)$ \\
\hline Dentin etching time & & 0.07 & & 0.06 & & \\
\hline Recommended by manufacturer (15s) & 1 & & 1 & & $36(78.3)$ & $10(21.7)$ \\
\hline Reduced etching time $(7 s)$ & $0.36(0.11-1.14)$ & & $0.35(0.11-1.12)$ & & $46(92.0)$ & $4(8.0)$ \\
\hline
\end{tabular}


Table 3. Evaluation of the restorations according to the FDI criteria used in this study.

\begin{tabular}{lccc}
\hline \multirow{2}{*}{$\begin{array}{l}\text { General evaluated } \\
\text { criteria }\end{array}$} & Specific evaluated criteria & Dentin etching time $(15 \mathrm{~s})$ & Dentin etching time $(7 \mathrm{~s})$ \\
\cline { 3 - 4 } Esthetic properties & Marginal staining & Restoration within each score $(1 / 2 / 3 / 4 / 5)^{*}$ & Restoration within each score $(1 / 2 / 3 / 4 / 5)^{*}$ \\
Functional properties & Fracture & $32 / 9 / 3 / 2 / 0$ & $42 / 8 / 0 / 0 / 0$ \\
Biological properties & Marginal adaptation & $41 / 2 / 0 / 1 / 2$ & $47 / 2 / 0 / 1 / 0$ \\
& Caries recurrence & $35 / 3 / 4 / 1 / 3$ & $41 / 4 / 3 / 2 / 0$ \\
\hline
\end{tabular}

*Numbers separated by slash represent the number of evaluated restorations for each score, according to the FDI criteria: 1 . Clinically excellent; 2. Clinically good; 3 . Clinically sufficient / satisfactory; 4 . Clinically unsatisfactory; 5 . Clinically poor.

sound and carious primary dentin. ${ }^{14}$ To the best of our knowledge, this is the first double-blind randomized clinical trial that assessed the effect of reduced acid etching time for dentin on restoration survival after selective carious tissue removal in primary molars.

A satisfactory survival rate of resin composite restorations was achieved during the 18 months follow-up period, irrespective of the dentin acid etching time. Estimated survival rates of the restorations performed with the shortening of the acid etching time were $100 \%, 98 \%, 96 \%$ and $91.4 \%$ at one, 6,12 and 18 months of evaluations, while the restorations performed with etching time recommended by manufacturer reached survival rates of $100 \%, 97.8 \%, 93.4 \%$, and $75.7 \%$ in the respective follow-up periods (Figure 2). A previous study ${ }^{20}$ found a survival rate at 18 months of $63.6 \%$ for resin composite restorations performed with $15 \mathrm{~s}$ of acid etching after selective carious tissue removal in primary molars. In addition, a university-based retrospective study ${ }^{21}$ reported an ARF of $18.8 \%$ of resin composite restorations placed in high caries-risk children, following the same adhesive protocol.

The etching time did not influence the clinical behavior of adhesive restorations $(p=0.06)$. However, we can observe a difference in the survival rates of $15.7 \%$ between treatment groups, with an AFR approximately 3 times lower when restorations were bonded after reduced etching time (AFR: $16.9 \%$ and $5.7 \%$ for 15 and $7 \mathrm{~s}$ ). Thus, even though this difference was statistically non-significant, it may still be clinically important. ${ }^{22}$ Despite there are currently no standardized measures of clinical significance, it is relevant to consider endpoints such as diminishing or simplification of future interventions and treatment costs.

In our study, specific parameters related to the adhesive protocol were considered for evaluation

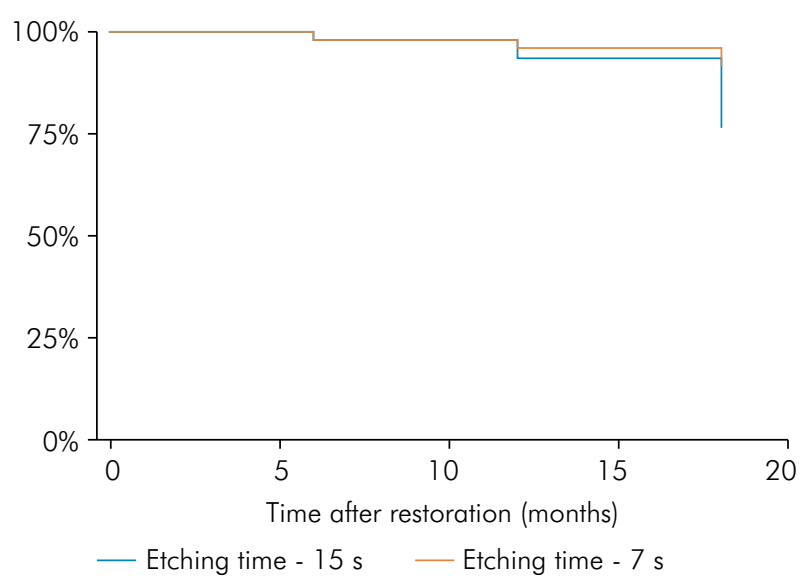

Figure 2. Survival curves (Kaplan-Meier) for $7 \mathrm{~s}$ and $15 \mathrm{~s}$ of acid etching over 18 months (log-rank $\mathrm{p}=0.06$ ).

of the restorations based on FDI criteria, ${ }^{16}$ including marginal staining, fracture associated with loss of restoration, marginal adaptation, and caries recurrence. FDI criteria ${ }^{16}$ classify failed restorations in two categories: whether the restoration can be corrected/repaired (score 4) and whether it must be replaced entirely (score 5). The main reasons for failure of restorations were marginal adaptation and fracture for both treatment groups. Nevertheless, it is important to highlight that all restorations placed after reduced etching time were repaired rather than replaced (Table 3). Furthermore, failures due to marginal staining occurred only when dentin was acid etched as per the time recommended by the manufacturer (15 s).

The tendency towards a higher risk of marginal discrepancies of restorations bonded after $15 \mathrm{~s}$ of acid etching may be related to higher discrepancy between demineralization and infiltration of resin monomers. Marginal defects are usually associated with deposits 
of food stains or bacterial biofilm into the marginal irregularities, such as gaps and micro- fractures, causing marginal staining. ${ }^{23}$ Therefore, shortening of the acid etching time could promote better adhesion in primary teeth, minimizing the occurrence or the severity of functional and esthetics failures.

The cavities were filled with a bulk-fill resin composite. Bulk-fill materials have the advantage of reducing chair-time, which is in line with the clinician's desire for simplified and fast procedures, and the number of steps for cavity restorations, which makes this procedure less prone to technical errors. It has been evidenced similar clinical performances of bulk-fill and conventional resin composites in posterior teeth. ${ }^{24} \mathrm{In}$ addition, no significant differences in the marginal gap formation and marginal integrity failures were found between resin composites. ${ }^{25}$ Therefore, we assumed that the functional and esthetics failures observed in our study were related to etching time for dentin.

Moreover, only occlusal cavities were included in our study to avoid the influence of other variables on the outcome. A reduced survival of occluso-proximal restorations in primary molars than occlusal restorations has been reported. ${ }^{15}$ Large proximal cavities result in preparations with boxes with limited retention due to the shape of primary molars. Failures were not also associated with individual and tooth-level variables such as gender, tooth type (first/second molar), arch (upper/lower), presence of gingival bleeding, or visible plaque at the restoration site.

The sample consisted of 100 primary molars from 62 children who had undergone restorative procedures. These patients belonged to a low socioeconomic level and were at a high caries-risk. It was expected that children included in the sample were more likely to experience restorative failures. ${ }^{25}$ Nonetheless, all children were included in a periodic recall program at the University, which explains the low dropout and few failures due to caries. Survival rate was calculated using the Kaplan-Meier estimator, which measures the fraction of the restorations surviving after the follow-ups. The estimator takes into account the censored data (i.e., restorations that were lost from the sample before the outcome occurred, exfoliated teeth, and/or those restorations not assessed in the 18-month evaluation), which can underestimate the real success rate of the restorative procedures during this period. Nevertheless, a high estimated survival rate $(84 \%)$ was found in this study.

Finally, we should mention the limitations of the study. The whole study was performed in a university setting, in which restorations were placed under ideal conditions with a calibrated operator to produce restorations as near perfect as possible. Although study design results in high internal validity, it could not reflect conditions closer to clinical practice.

Shortening of the acid etching time for dentin by half the recommended by manufacturer $(7 \mathrm{~s})$ is a simple strategy and clinically applicable for minimizing the discrepancy between the depth of demineralization and infiltration of the resin monomers. Since there was a tendency for better clinical outcome (not statistically significant, but clinically relevant) with etching time reduced to approximately half of the manufacturer's recommended time, this new adhesive protocol for etch-and-rinse systems could be used in pediatric dentistry (enamel etching time remains $15 \mathrm{~s}$ ).

\section{Acknowledgements}

This study was financed in part by the Coordenação de Aperfeiçoamento de Pessoal de Nível Superior Brasil (CAPES) - Finance Code 001 and Conselho Nacional de Desenvolvimento Cientifico e Tecncologico (CNPq) grant 302192/2018-2. We would like to thank Pablo Silveira dos Santos for support with treatment of other needs of children included in the study.

\section{References}

1. Pedrotti D, Ribeiro JF, Pires CW, Rocha RO, Ardenghi TM, Soares FZ, et al. Survival and associated risk factors of resin-based composite restorations in primary teeth: a clinical, retrospective, university-based study. Pediatr Dent. 2017 Jul;39(4):313-8.

2. Pinto GS, Oliveira LJ, Romano AR, Schardosim LR, Bonow ML, Pacce M, et al. Longevity of posterior restorations in primary teeth: results from a paediatric dental clinic. J Dent. 2014 Oct;42(10):1248-54. https://doi.org/10.1016/i.jdent.2014.08.005 
3. Bücher K, Metz I, Pitchika V, Hickel R, Kühnisch J. Survival characteristics of composite restorations in primary teeth. Clin Oral Investig. 2015 Sep;19(7):1653-62. https://doi.org/10.1007/s00784-014-1389-9

4. Campagna P, Pinto LT, Lenzi TL, Ardenghi TM, Rocha RO, Oliveira MD. Survival and associated risk factors of composite restorations in children with early childhood caries: a clinical retrospective study. Pediatr Dent. 2018 May;40(3):210-4.

5. Ribeiro JF, Forgerini TV, Pedrotti D, Rocha RO, Ardenghi TM, Soares FZ, et al. Performance of resin composite restorations in the primary dentition: a retrospective university-based study. Int J Paediatr Dent. 2018 Jul;28(5):497-503. https://doi.org/10.1111/ipd.12404

6. Demarco FF, Collares K, Correa MB, Cenci MS, Moraes RR, Opdam NJ. Should my composite restorations last forever? Why are they failing? Braz Oral Res. 2017 Aug;31 suppl 1:e56. https://doi.org/10.1590/1807-3107bor-2017.vol31.0056

7. Lenzi TL, Gimenez T, Tedesco TK, Mendes FM, Rocha RO, Raggio DP. Adhesive systems for restoring primary teeth: a systematic review and meta-analysis of in vitro studies. Int J Paediatr Dent. 2016 Sep;26(5):364-75. https://doi.org/10.1111/ipd.12210

8. Angker L, Nockolds C, Swain MV, Kilpatrick N. Quantitative analysis of the mineral content of sound and carious primary dentine using BSE imaging. Arch Oral Biol. 2004 Feb;49(2):99-107. https://doi.org/10.1016/i.archoralbio.2003.08.006

9. Lenzi TL, Guglielmi CA, Arana-Chavez VE, Raggio DP. Tubule density and diameter in coronal dentin from primary and permanent human teeth. Microsc Microanal. 2013 Dec;19(6):1445-9. https://doi.org/10.1017/S1431927613012725

10. Nör JE, Feigal RJ, Dennison JB, Edwards CA. Dentin bonding: SEM comparison of the resin-dentin interface in primary and permanent teeth. J Dent Res. 1996 Jun;75(6):1396-403. https://doi.org/10.1177/00220345960750061101

11. Senawongse P, Harnirattisai C, Shimada Y, Tagami J. Effective bond strength of current adhesive systems on deciduous and permanent dentin. Oper Dent. 2004 Mar-Apr;29(2):196-202.

12. Yoshiyama M, Tay FR, Doi J, Nishitani Y, Yamada T, Itou K, et al. Bonding of self-etch and total-etch adhesives to carious dentin. J Dent Res. 2002 Aug;81(8):556-60. https://doi.org/10.1177/154405910208100811

13. Haj-Ali R, Walker M, Williams K, Wang Y, Spencer P. Histomorphologic characterization of noncarious and caries-affected dentin/adhesive interfaces. J Prosthodont. 2006 Mar-Apr;15(2):82-8. https://doi.org/10.1111/j.1532-849X.2006.00079.x

14. Lenzi TL, Braga MM, Raggio DP. Shortening the etching time for etch-and-rinse adhesives increases the bond stability to simulated cariesaffected primary dentin. J Adhes Dent. 2014 Jun;16(3):235-41.

15. Franzon R, Opdam NJ, Guimarães LF, Demarco FF, Casagrande L, Haas AN, et al. Randomized controlled clinical trial of the 24 -months survival of composite resin restorations after one-step incomplete and complete excavation on primary teeth. J Dent. 2015 Oct;43(10):1235-41. https://doi.org/10.1016/i.ident.2015.07.011

16. Hickel R, Peschke A, Tyas M, Miör I, Bayne S, Peters M, et al. FDI World Dental Federation - clinical criteria for the evaluation of direct and indirect restorations. Update and clinical examples. J Adhes Dent. 2010 Aug;12(4):259-72. https://doi.org/10.1007/s00784-010-0432-8

17. Schwendicke F, Frencken JE, Biørndal L, Maltz M, Manton DJ, Ricketts D, et al. Managing Carious Lesions: Consensus Recommendations on Carious Tissue Removal. Adv Dent Res. 2016 May;28(2):58-67. https://doi.org/10.1177/0022034516639271

18. Nakajima M, Sano H, Burrow MF, Tagami J, Yoshiyama M, Ebisu S, et al. Tensile bond strength and SEM evaluation of caries-affected dentin using dentin adhesives. J Dent Res. 1995 Oct;74(10):1679-88. https://doi.org/10.1177/00220345950740100901

19. Lenzi TL, Mendes FM, Rocha RO, Raggio DP. Effect of shortening the etching time on bonding to sound and caries-affected dentin of primary teeth. Pediatr Dent. 2013 Sep-Oct;35(5):E129-33.

20. Casagrande L, Dalpian DM, Ardenghi TM, Zanatta FB, Balbinot CE, García-Godoy F, et al. Randomized clinical trial of adhesive restorations in primary molars. 18-month results. Am J Dent. 2013 Dec;26(6):351-5.

21. Dalpian DM, Gallina CS, Nicoloso GF, Correa MB, Garcia-Godoy F, Araujo FB, et al. Patient- and treatment-related factors may influence the longevity of primary teeth restorations in high caries-risk children: a university-based retrospective study. Am J Dent. 2018 Oct;31(5):261-6.

22. Houle TT, Stump DA. Statistical significance versus clinical significance. Semin Cardiothorac Vasc Anesth. 2008 Mar;12(1):5-6. https://doi.org/10.1177/1089253208316440

23. Heintze SD, Rousson V. Clinical effectiveness of direct class II restorations - a meta-analysis. J Adhes Dent. 2012 Aug;14(5):407-31.

24. Veloso SR, Lemos CA, Moraes SL, Vasconcelos BCE, Pellizzer EP, Monteiro GQM. Clinical performance of bulk-fill and conventional resin composite restorations in posterior teeth: a systematic review and meta-analysis. Clin Oral Investig. 2019 Jan;23(1):221-33. https://doi.org/10.1007/s00784-018-2429-7

25. Loguercio AD, Rezende M, Gutierrez MF, Costa TF, Armas-Vega A, Reis A. Randomized 36-month follow-up of posterior bulk-filled resin composite restorations. J Dent. 2019 Jun;85:93-102. https://doi.org/10.1016/i.ident.2019.05.018

26. Correa MB, Peres MA, Peres KG, Horta BL, Barros AJ, Demarco FF. Do socioeconomic determinants affect the quality of posterior dental restorations? A multilevel approach. J Dent. 2013 Nov;41(11):960-7. https://doi.org/10.1016/i.jdent.2013.02.010 